\title{
Reverberation Mapping Results from MDM Observatory
}

\author{
Kelly D. Denney ${ }^{1}$, B. M. Peterson ${ }^{1}$, R. W. Pogge ${ }^{1}$, M. C. Bentz ${ }^{2}$, \\ C. M. Gaskell ${ }^{3}$, T. Minezaki ${ }^{4}$, C. A. Onken ${ }^{5}$, S. G. Sergeev ${ }^{6}$, and \\ M. Vestergaard ${ }^{7}$ \\ ${ }^{1}$ The Ohio State University, 140 West 18th Avenue, Columbus, OH 43210, USA \\ Email: denney@astronomy.ohio-state.edu \\ ${ }^{2}$ University of California at Irvine, USA \\ ${ }^{3}$ University of Texas at Austin, USA \\ ${ }^{4}$ University of Tokyo, Japan \\ ${ }^{5}$ Mount Stromlo Observatory, Australia \\ ${ }^{6}$ Crimean Astrophysical Observatory, Ukraine, and Isaak Newton Institute, Chile \\ ${ }^{7}$ University of Copenhagen, Denmark
}

Keywords. black hole physics, galaxies: active, galaxies: Seyfert

Reverberation mapping takes advantage of the presence of a time delay or lag, $\tau$, between continuum and emission line flux variations observed through spectroscopic monitoring campaigns to infer the radius of the broad-line region (BLR) and, subsequently, the central black hole mass in type 1 AGNs. We present results from a multi-month reverberation mapping campaign undertaken primarily at MDM Observatory with supporting observations from around the world. We measure BLR radii and black hole masses for six objects. The primary goal of this campaign was to obtain either new or improved $\mathrm{H} \beta$ reverberation lag measurements for several relatively low-luminosity AGNs. Using cross correlation techniques to measure the time delay between the mean optical continuum flux density around $5100 \AA$ and the integrated $\mathrm{H} \beta$ flux, we determine the $\mathrm{H} \beta$ lags and black hole mass measurements listed in columns 2 and 3 of Table 1, respectively. Column 4 tells if this measurement is new, an improvement meant to replace a previous, less reliable measurement, or simply an additional measurement not used to replace a previous value. The complete results from this study are currently being prepared for publication. A subsequent velocity-resolved analysis of the $\mathrm{H} \beta$ response shows that three of the six primary targets demonstrate kinematic signatures (column 5) of infall, outflow, and non-radial virialized motions (see Denney et al. 2009).

Table 1. Mean H $\beta$ Lags and Black Hole Masses

\begin{tabular}{lcccc}
\hline Object & $\tau_{\text {cent }}$ (days) & $M_{\mathrm{BH}}\left(\times 10^{6} M_{\odot}\right)$ & Data Use & Kinematic Signature \\
\hline NGC 3227 & $3.75_{-0.82}^{+0.76}$ & $7.63_{-1.72}^{+1.62}$ & improvement & infall \\
NGC 3516 & $11.68_{-1.53}^{+1.02}$ & $31.7_{-4.8}^{+2.8}$ & improvement & outflow \\
NGC 5548 & $12.40_{-3.85}^{+2.74}$ & $44.2_{-13.8}^{+9.9}$ & add'l measurement & virial \\
Mrk 290 & $8.72_{-1.02}^{+1.21}$ & $24.3_{-3.7}^{+3.7}$ & new & - \\
Mrk 817 & $14.04_{-3.47}^{+3.41}$ & $61.9_{-15.3}^{+15.0}$ & add'l measurement & - \\
NGC 4051 & $1.87_{-0.50}^{+0.54}$ & $1.73_{-0.52}^{+0.55}$ & improvement & - \\
\hline
\end{tabular}

\section{Reference}

Denney, K. D., et al. 2009, ApJ, 704, L80 